\title{
Utilization of Teledentistry in Antimicrobial Prescribing and Diagnosis of Infectious Diseases during COVID-19 Lockdown
}

\author{
Najla Dar-Odeh ${ }^{1,2}$ Hamzah Babkair ${ }^{1} \quad$ Ahmad Alnazzawi ${ }^{3}$ Shaden Abu-Hammad² \\ Abdalla Abu-Hammad ${ }^{4}$ Osama Abu-Hammad ${ }^{2,3}$
}

${ }^{1}$ Department of Oral Basic and Clinical Sciences, College of Dentistry, Taibah University, Al Madinah Al Munawwarah, Saudi Arabia

2Department of Oral Surgery, Oral Medicine and Periodontics, School of Dentistry, University of Jordan, Amman, Jordan

${ }^{3}$ Department of Substitutive Dental, College of Dentistry, Taibah University, Al Madinah Al Munawwarah, Saudi Arabia

${ }^{4}$ School of Medicine, University of Jordan, Amman, Jordan

\begin{abstract}
Address for correspondence Osama Abu-hammad, PhD, Department of Substitutive Dental Sciences, College of Dentistry, Taibah University, Al Madinah Al Munawwarah 43353, Saudi Arabia (e-mail: o.abuhammad@yahoo.com).
\end{abstract}

Eur J Dent:2020;14(suppl S1):S20-S26

\begin{abstract}
Keywords

- antibiotic prescribing

- COVID-19

- dentists

- diagnosis

- oral infections

- social media
\end{abstract}

Objectives This study aimed to analyze Jordanian dentists' inquiries on oral infections and antimicrobial prescribing using dental professional WhatsApp groups during coronavirus disease lockdown period.

Materials and Methods Three professional WhatsApp groups of Jordanian dentists were reviewed and analyzed for inquiries posted during the period from January to May 2020. Inquiries were sent from patients to their dentists who posted these inquiries to the professional WhatsApp dental groups for consultation and professional advice. All queries regarding oral infections and antimicrobial prescribing were extracted and analyzed.

Results Three WhatsApp professional groups with a total numbers of members of 750 dentists who posted queries about their patients were included in this study. Dentist members posted queries about their patients to these professional WhatsApp groups. There was a total of 32 inquiries regarding oral lesions and 11 consultations regarding prescribing and dental management of medically compromised patients giving a total of 43 consultations. Among which there were 19 inquiries on oral infections and 9 inquiries on antimicrobial prescribing giving a total of 28 consultations. Most common inquiries were on bacterial infections (localized dentoalveolar abscess, pericoronitis, cellulitis, and lymphoid hyperplasia of the tongue), viral herpetic infections, and Candida infections (erythematous and pseudomembranous candidiasis).

Conclusions Many dental practitioners cannot distinguish the correct diagnostic features of oral infections particularly viral and fungal infections. Continuing education should be considered to focus on clinical manifestations of various oral infections. Further, educational activities that focus on variations in treatment protocols for various infections should be introduced particularly those that concern indications for antimicrobial prescribing.
DOI https://doi.org/

10.1055/s-0040-1717159

ISSN 1305-7456. (c) 2020. European Journal of Dentistry.

This is an open access article published by Thieme under the terms of the Creative Commons Attribution-NonDerivative-NonCommercial-License, permitting copying and reproduction so long as the original work is given appropriate credit. Contents may not be used for commercial purposes, or adapted, remixed, transformed or built upon. (https://creativecommons.org/licenses/by-nc-nd/4.0/)

Thieme Medical and Scientific Publishers Pvt. Ltd., A-12, 2nd Floor, Sector 2, Noida-201301 UP, India 


\section{Introduction}

Coronavirus disease (COVID-19) was declared a pandemic by the World Health Organization in early 2020, with substantive numbers of cases and deaths reported in many countries. ${ }^{1}$ As of May 22, 2020, there were more than 5 million cases and more than 340,000 deaths globally. While the novel virus causing this infection was identified as severe acute respiratory syndrome coronavirus 2 (SARS-CoV-2) or novel COVID-19, clinical manifestations were described to affect the respiratory system, as well as skin, nervous, cardiovascular, and gastrointestinal systems among others. ${ }^{2}$

The emergence of COVID-19 pandemic has had a great impact on health systems in many countries and dental practice is no exception. Jordan was one of the countries that were influenced greatly by the pandemic. While the first cases of this infection have been identified in Wuhan, China late November 2019, it was not until early March that the first case of COVID-19 was identified in Jordan in a national who arrived from Italy few weeks before. The reaction from Jordanian government was swift, and strict, and lockdown measures were initiated later on March 17. In line with recommendations of the American Dental Association, ${ }^{3}$ and lockdown announced by the government, the Jordanian Dental Association has called upon all dental practices to stop provision of all types of dental treatment. It was obvious that the main priority of the Ministry of Health was to stop the spread of COVID-19 infection and to provide treatment to those infected. Later, at the beginning of May, the lockdown was partially eased and dental practices were allowed to return to work to provide emergency dental treatment under certain strict working conditions.

It was evident during this critical time of lockdown that teledentistry is the most effective way for dental practitioners to communicate their concerns as well as their professional queries. ${ }^{4}$ Teledentistry allows dentists to utilize telecommunications to share and exchange clinical information and images over remote distances, hence facilitating dental consultations regarding diagnosis and treatment planning. Benefits of teledentistry include enhancing dentists' expertise, and obtaining advice for difficult clinical situations. ${ }^{5}$ One of the most important aspects of oral health care that can be communicated over social media during lockdown is the management of acute oral infections and prescribing of antimicrobials. Antimicrobial prescribing has always been considered a significant aspect of dental practice, ${ }^{6}$ with the majority of drugs prescribed by dentists being antimicrobials. ${ }^{7}$ Prescribing of antimicrobials is not without a cost especially that dentists in many countries showed a tendency toward overprescribing and using broad-spectrum antibiotics. ${ }^{8}$ They also were inclined to prescribe for nonindicated clinical conditions. ${ }^{9}$ Development of antimicrobial resistance and many complications and side effects associated with antimicrobial use are consequently anticipated. ${ }^{10}$

A substantial proportion of oral infections have an acute nature, and their diagnosis and management should be done promptly. During lockdown, WhatsApp professional dental groups proved to be a very effective way for dentists to consult their colleagues about the complaints of their patients particularly those concerned with infections. ${ }^{11}$ This study aimed to analyze Jordanian dentists' consultations on oral infections and antimicrobial prescribing using dental professional WhatsApp groups, during the period from January to May 2020. During this period, COVID-19 was identified, and declared as a pandemic with implementation of lockdown involving dental practices.

\section{Materials and Methods}

Three professional WhatsApp groups of Jordanian dentists (WhatsApp Messenger, WhatsApp Inc., Mountain View, California, United States) were reviewed and analyzed for queries posted during the period from January to May 2020. All queries pertaining to oral medicine, oral diseases, dental prescribing, and dental precautions in medically compromised patients were extracted. Queries (questions and images) on oral infections and antimicrobial prescribing were examined. The following data were collected: onset and duration of lesions, clinical manifestations, associated symptoms (where applicable), and age and gender of patients (where applicable). Infections were categorized into three types: bacterial and protozoal, viral, and Candida infections.

\section{Results}

Three professional WhatsApp groups of Jordanian dentists with $\sim 750$ members were included in this study.

During the period from January to May 2020, there were a total of 32 consultations regarding oral diseases and 11 consultations regarding prescribing and dental management of medically compromised patients. This resulted in a total of 43 consultations on oral diseases, prescribing and dental management of medically compromised patients.

Among these, there were 19 consultations regarding diagnosis and management of oral infections, and 9 consultations on prescribing giving a total of 28 consultations.

Consultations on oral infections were distributed as follows:

1. Bacterial and protozoal infections: facial cellulitis (three cases), localized dentoalveolar abscess (two cases), pericoronitis (one case), lymphoid hyperplasia of the tongue (two cases), and suspected leishmaniasis of the lip (one case) ( - Figs. 1 and $\mathbf{2}$ ).

2. Viral infections: hand, foot, and mouth disease (one case), intraoral herpes infection (one case) (-Fig. 2), acute herpetic gingivostomatitis (three cases), and recurrent herpes labialis (three cases) (-Figs. 3 and 4 ).

3. Oral candidiasis: one case each (acute erythematous candidiasis on tongue and palate of 25-year-old woman, and pseudomembranous candidiasis in a female infant with a history of a broad-spectrum antibiotic intake for otitis media) (-Fig. 5).

There were also nine inquiries on antibiotic prescribing distributed as follows: 


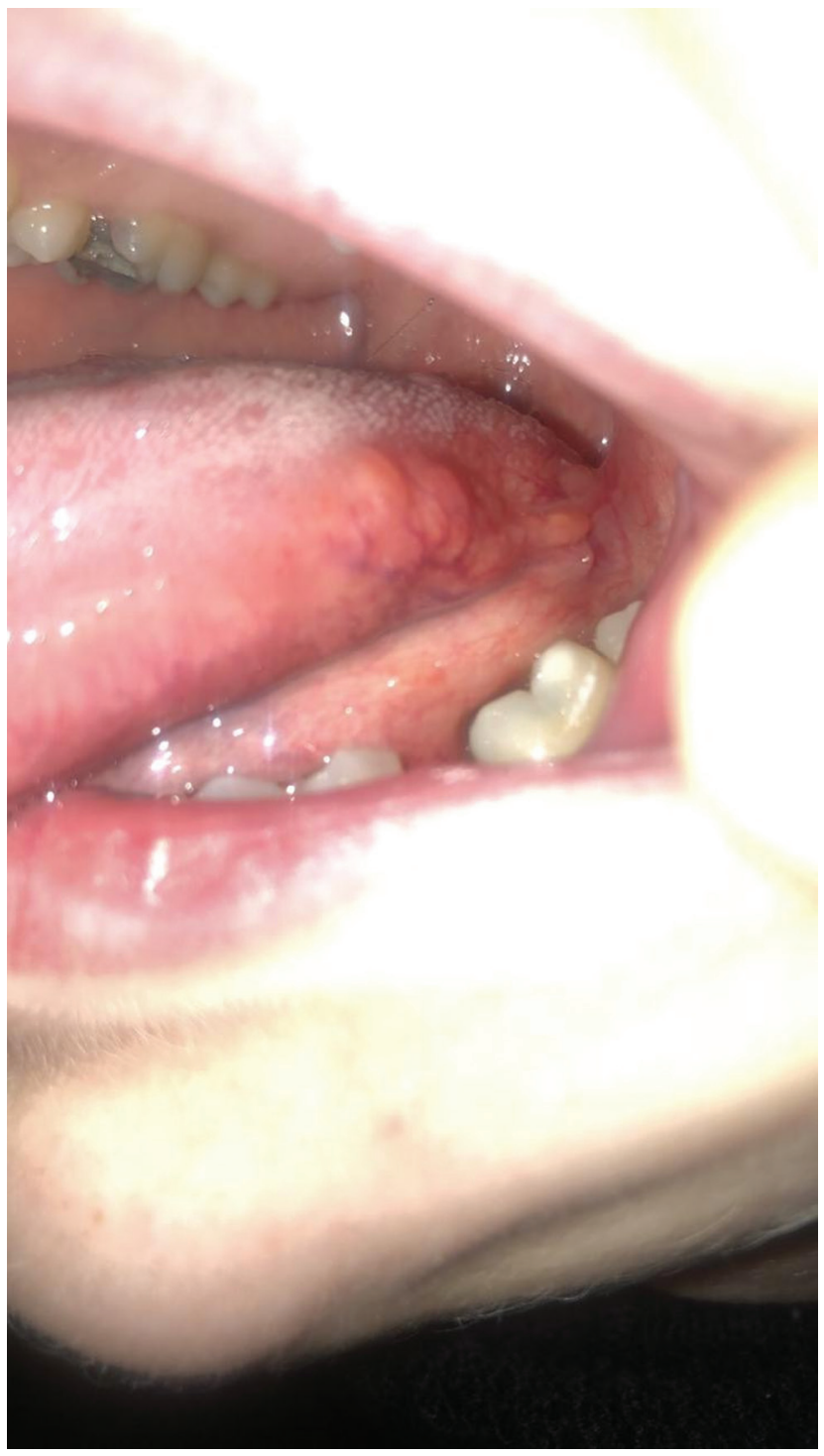

Fig. 1 A young female with lymphoid hyperplasia of the tongue. The lesion is bilateral with occasional swelling and inflammation that responds to improved oral hygiene and topical antiseptic therapy.

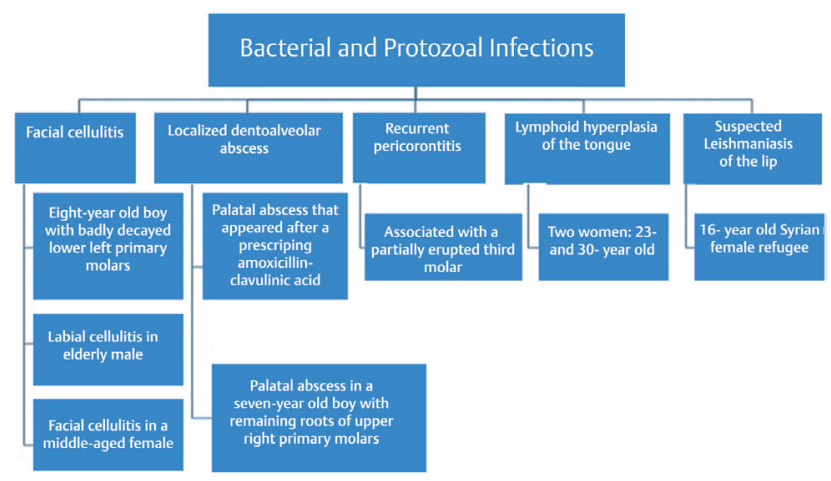

Fig. 2 Description of bacterial and protozoal infections. Age and gender were not available for all patients.

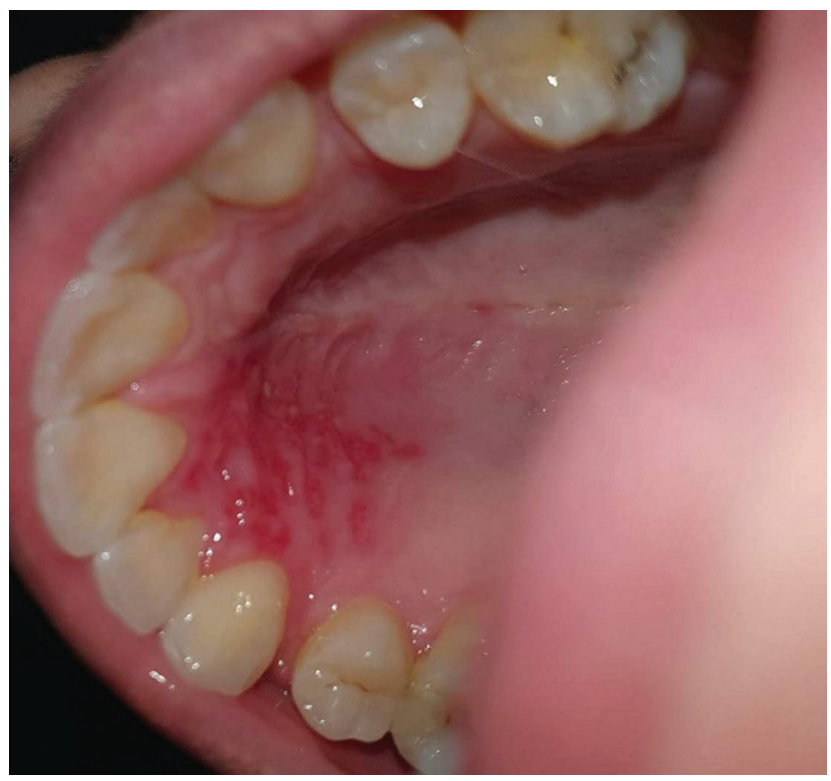

Fig. 3 Intraoral pinpoint ulcers affecting the anatomic region innervated by the right nasopalatine nerve in a young patient. Shape, pattern of distribution, and acute onset suggest a viral etiology.

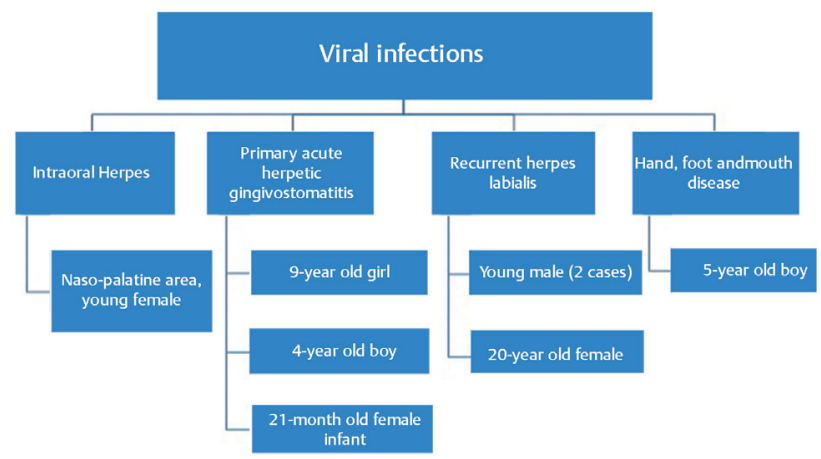

Fig. 4 Types of orofacial viral infections describing age and gender distribution.

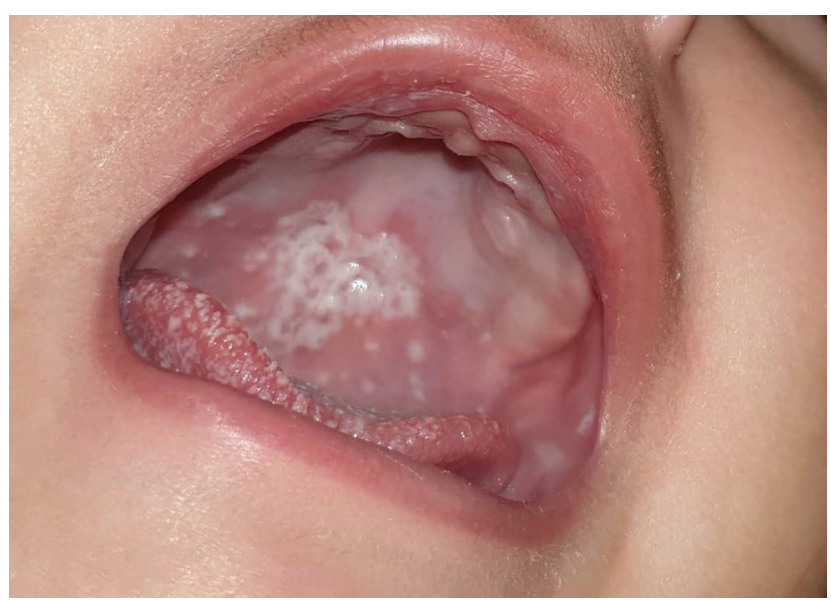

Fig. 5 Pseudomembranous candidiasis (thrush) in an infant who received a broad-spectrum antibiotic few days previously. White patches can be wiped off to reveal a red inflamed underlying mucosa. 
1. The recommended antibiotic for a patient with gingivitis.

2. Suitability of prescribing metronidazole on the request of a diabetic patient's son, and as an alternative to amoxicillin.

3. The need for prescribing antibiotic to a diabetic patient with blood sugar of 180 , severe pain around wisdom tooth indicated for extraction.

4. Suitability of prescribing clindamycin to a breastfeeding mother, with facial cellulitis, that was treated in the previous month with amoxicillin-clavulanic acid.

5. Consultation regarding diagnosis and management for a patient who was treated with cefixime for facial cellulitis with no success, and later developed oral ulcers similar to the pattern of erythema multiforme. As a replacement, the patient continued on amoxicillin-clavulanic acid and metronidazole.

6. Suitability of lincomycin for treatment of facial cellulitis.

7. What is the best antibiotic for the dental patient?

8. What is the recommended antibiotic for patients allergic to penicillin?

9. What are the precautions for an endocarditis patient who needs extraction?

\section{Discussion}

Jordan is a small Middle Eastern country populated by $\sim 10$ million people. Health system in the kingdom has shown remarkable efficiency to contain the COVID-19 pandemic since the identification of the first patient diagnosed with the disease in the country and this has greatly minimized the severe adverse outcomes of the disease. ${ }^{12}$ As of May 22, 2020, there were 700 cases, among whom 461 recovered and 9 have deceased. Strict measures conducted by the government involved partial and complete lockdown that were implemented according to the current pandemic situation taking into account the basic needs of the society. Dental practices were included in the lockdown, and all dental practices were closed. It was practical for dentists to utilize social media to communicate with their patients as well as their colleagues to convey their patients' complaints, and their professional queries and concerns. It was evident from the results of this study that oral infections were the most common patients' complaints, and that queries on antibiotics were the most commonly communicated topic among dentists. Jordan has been characterized by being one of the most politically stable and medically advanced Middle Eastern countries. However, oral medicine specialty has remained underrepresented by a limited number of specialists who mainly work in academic and public health institutions, namely, public universities and the Ministry of Health hospitals situated in the capital and major cities. Establishment of several professional dental groups on WhatsApp application has facilitated communications among Jordanian dentists whether those practicing inside Jordan or abroad.

Most consultations regarding prescribing were concerned with diagnosis of oral infections that were mostly acute in nature. Other important consultations were on antibiotics, and these included some questions that all dentists should be familiar with, such as "recommended antibiotics for dental infection" and "alternative antibiotics in patients allergic to penicillin." Another example was the consultation regarding lincomycin prescription to patients with facial cellulitis. Facial cellulitis is an acute bacterial infection that is diagnosed by identifying its clinical manifestations of a unilateral acute swelling of the face associated usually with an infected tooth. The primary focus of infection is usually identified as an abscess around the tooth apex. It is important to accurately diagnose facial cellulitis as well as identify the tooth with the primary source of infection. Facial cellulitis is one of the few indications for antibiotic prescribing in dentistry. Lincomycin is a naturally occurring lincosamide produced by a Streptomyces species, and it has a high activity against anaerobic bacteria. Although lincomycin has a good antistaphylococcal and antistreptococcal activity, ${ }^{13}$ it is not among the antibiotics recommended in dental infections. In the most recent publication by the American Association of Endodontists on antibiotic prescribing, azithromycin, cephalexin, and clindamycin were recommended as alternatives to amoxicillin in penicillin-allergic patients. ${ }^{14}$ Lincomycin in clinical practice has been replaced by the more effective and safer lincosamide, clindamycin. Therefore, its use in dental practice should not be encouraged.

Unfortunately, the correct indications for antibiotic prescribing are still unknown to some practitioners, as some of them considered that gingivitis may be treated by antibiotics. While plaque-induced gingivitis is treated by physical plaque and calculus removal, a limited number of periodontal diseases are indicated for antibiotics, namely, necrotizing ulcerative gingivitis and pericoronitis. ${ }^{9}$ Pericoronitis treatment is dependent on the severity of infection and recurrence whereby options include antibiotics prescription, operculotomy, and extraction if the infection is recurrent. ${ }^{15}$ It was also obvious that nonclinical or social factors still influence prescribing as shown by the case of the son of an elderly patient with diabetes and stents who requested a metronidazole prescription from the dentist. The dentist was willing to write a prescription without physically examining the patient and was consulting the colleagues regarding whether amoxicillin should be alternatively prescribed. "Social" prescribing was found to be a common attitude among dentists in several countries particularly from the developing world. ${ }^{16}$ Unfortunately, many physicians and dentists extend cultural attitudes of generosity toward their patients by prescribing so many medications, most of them may be unnecessary or even contraindicated. ${ }^{17}$

Insufficient knowledge and leniency in antibiotic prescribing were shown in the case of the breastfeeding mother with facial cellulitis for which she kept taking antibiotics rather than receiving the proper operative dental treatment to remove the cause of infection. The patient had an episode of facial cellulitis treated by amoxicillin, and within few weeks, she had another episode of facial cellulitis that necessitated treatment with a different antibiotic. It was not clear why the proper dental treatment was postponed and patient kept receiving antibiotic prescriptions, although lockdown was not yet activated at the time. This may indicate poor attitude toward dental attendance and oral health care behavior. 
Indeed, it was shown that pregnant and breastfeeding mothers of the Middle East have a tendency to avoid dental visits, mainly because of their concerns for the safety of pregnancy, and safety of infant thinking that dental treatment may pose a threat to the health of mother or fetus. ${ }^{18,19}$ Dentists have a commitment toward their patients by motivating them to pursue oral health care and by educating them regarding the inappropriate use of antibiotics. Breastfeeding mothers and pregnant women constitute an important category of vulnerable patients for which dental prescribing should be clear and concise. The guidelines are clear for prescribing during lactation and pregnancy. Any drug prescribed during pregnancy can cross placenta depending on their lipid solubility and molecular weight. For most drugs, the infant is exposed to a much higher concentration during pregnancy than during lactation. Therefore, if a drug is considered acceptable for use during pregnancy, it is usually reasonable to use it during breastfeeding. ${ }^{20}$ Drugs should be prescribed in pregnancy only if the expected benefit to the mother is thought to be greater than the risk to the fetus, while use of all drugs should be avoided if possible during the first trimester. ${ }^{21}$ Several antibiotics are considered safe in pregnancy and breastfeeding including amoxicillin, azithromycin, and clindamycin. Metronidazole is a broad-spectrum antibiotic that is effective against anaerobic bacteria and protozoa, ${ }^{22}$ and although it is safe in pregnancy, its use in breastfeeding is not recommended because it may affect the taste of milk adversely. ${ }^{21}$

Within the context of vulnerable populations, there were two types of infections affecting infants younger than 2 years: acute herpetic gingivostomatitis and pseudomembranous candidiasis. The former is a viral infection likely to affect young children, and its diagnosis is mainly achieved by means of the associated clinical manifestations of widespread intraoral ulcers that may involve the gingiva, fever, and cervical lymphadenopathy. It is important to achieve the correct diagnosis especially that worried parents may interpret this as an infection that necessitates an antibiotic infection, whereas it is a self-limiting viral infection. Management of oral viral infections particularly the herpes family of viruses is very important as these could be associated with chronic periodontitis. ${ }^{23}$ Pseudomembranous candidiasis, on the contrary, can be associated with several predisposing local and systemic factors. ${ }^{24}$ In this case, it was associated with the use of a broad-spectrum antibiotic. The use of broad-spectrum antibiotics in infants to treat respiratory tract infections may be associated with several adverse effects such as allergy, gastrointestinal disturbances, and candidiasis. ${ }^{25}$ These antibiotics can compromise bacterial microbiota levels and reduce colonization resistance leading to overgrowth of Candida species. ${ }^{26}$ Treatment in this situation involves stopping the predisposing antibiotic and administering a topical antifungal drug.

It was interesting to find two consultations on lymphoid hyperplasia of the tongue during this period. Both of them were in young female patients in the third decade of life. These lumps are normal anatomic structures in some patients and are usually asymptomatic. However, they may get inflamed and present with recurrent painful episodes affecting the posterolateral aspects of the tongue. ${ }^{27}$ Patients mainly seek treatment after noticing a mass on the tongue that may resemble a cancerous lesion. Therefore, it is important that the correct diagnosis is established based on the clinical appearance for these structures are considered anatomic variations that may get inflamed sometimes as any other lymphoid tissue. If the lesions are in otherwise asymptomatic patients, they should be reassured of the benign nature of the lesion. If they are associated with inflammation and pain, patients are instructed to have a good oral hygiene including tongue scraping and use of antiseptic mouthwash. In all cases, patients are followed up just to exclude the continuous growth typical of cancer.

Oral herpetic infections were also among dentists' consultations. The Herpes family of viruses causes numerous infections in the orofacial regions. The most common of these are caused by herpes simplex I and/or II. These DNA viruses cause acute infections that are manifested orally as pinpoint ulcers that may coalesce later on to become larger irregular ulcers. Although these ulcers are preceded by vesicular eruption, vesicles are rarely noticed by patients, or detected by dentists because unlike cutaneous lesions, oral lesions are fragile and they rupture promptly. In immunocompetent patients, the self-limiting nature of the infection excludes the need for antiviral therapy and necessitates the use of supportive therapy as early as possible. There were three cases of acute herpetic gingivostomatitis affecting patients with age range 2 to 9 years. The severity of the acute (primary) form and the young age of affected patients (children) usually cause concern among parents who may pressure the dentist to prescribe antibiotics. However, the aims of therapy should be the relief of pain, controlling fever, and preventing dehydration. Hence, treatment in the form of analgesic/anesthetic topical applications, oral analgesics/antipyretics, and maintaining sufficient fluid intake play an important role in recovery.

It was interesting to see that recurrent herpes labialis is still associated with confusion in diagnosis, although it is a common infection especially during winter season.

There were a few case reports published on COVID-19 as a possible cause of oral ulcers. ${ }^{28,29}$ However, these observations should be interpreted with caution since they occurred in patients who were suspected cases of COVID-19, and not yet diagnosed for having the disease. Further, other cases developed these ulcerative lesions following treatment of COVID-19 with immunosuppressive drugs and antibiotics. The clinical appearance of the reported lesions was very similar to intraoral herpetic infections. Most cases were diagnosed through the use of social media, and no intraoral clinical examination was done for patients. Moreover, no laboratory testing was done to exclude other viral causes of intraoral ulcers. The assumption that SARS-CoV-2 is the causative virus for these lesions may be coincidental and based on circumstantial evidence. It is true that the genome of SARSCoV-2 has been detected in saliva in the majority of patients with this disease, ${ }^{30}$ and in some cases, SARS-CoV-2 was detected only in saliva, with no evidence for its presence in 
the nasopharynx..$^{31}$ However, caution should be practiced when associating COVID-19 with oral ulcers as there are many viruses affecting the oral cavity, and several drugs used for treatment of COVID-19 could afflict the oral mucosa by ulcers. COVID-19 and its treatment are expected to compromise immunity and increase susceptibility to a wide range of opportunistic fungal and viral infections. Moreover, emotional stress associated with home quarantine, lockdown, financial worries, and infection of beloved friends and family members is also expected to jeopardize health and complicate the clinical picture.

It was not the aim of this study to analyze online workshops that were posted for dentists during the same period; however, it was noticed that there were more than 100 online workshops that were promoted through the included WhatsApp groups during the study period. All of these workshops discussed operative aspects of dentistry, such as restorative, esthetic, and implant dentistry, and none was concerned with oral infections or antibiotic prescribing.

Dentists and dental assistants are considered among the high-risk groups for COVID-19 infections. Many of them now are limiting the numbers of their patients until the pandemic situation is cleared. On the contrary, many patients are also hesitant to visit dental practices either because of their worries to contract infection, or because of the deteriorating financial condition associated with unemployment and deteriorating economy. Indeed, many dental practices have now increased dental visits fees, due to employment of more costly personal protective equipment to meet the increasing demands of cross infection control measures needed for COVID-19 prevention. All of these factors are likely to increase the dependence of dental patients on social media and hence make it a popular method for communication between dentists.

The study has limitations represented by the inclusion of only three professional groups; however, there were 750 members in these groups representing general practitioners as well as specialists. Nearly 6,000 dentists are now registered in Jordan, hence the study sample constituted $12.5 \%$ of the total number which is acceptable. The study was also retrospective in nature, so it is expected to demonstrate the actual situation of dentists' consultations.

\section{Conclusion}

During COVID-19 lockdown, most Jordanian dentists' queries in their WhatsApp professional groups were on oral infections and antibiotic prescribing. COVID-19 pandemic has not been over yet, so the increased dependence on social media for professional consultations either from patients or dentists is likely to continue for some time. It seems that most of dentists' consultations were on oral infections and antimicrobial prescribing. An important example is viral infections, the diagnosis of which may be confusing for some practitioners. These infections are mostly acute and transient in nature. Appearance of ulcers and associated symptoms of soreness, lymphadenopathy, and potential fever/malaise may cause concern among patients and/or their dentists. However, it is important to use accurate diagnostic criteria for these lesions so as to avoid faulty management, using antibiotics for example.

Although it is important to take the necessary measures to regulate the use of social media for this purpose so that dentists are protected medicolegally and financially, it should be emphasized that diagnosis of oral infections is mainly based on clinical features rather than laboratory investigations. In the case of bacterial infections such as facial cellulitis, treatment has two main goals: treatment of infection by prescribing an appropriate antibiotic, and identifying the source of infection by thorough clinical examination and radiographic investigations.

Continuing dental education should expand its learning outcomes to include not only money-generating operative dental procedures but also diagnosis of orofacial infections and appropriate indications for antibiotic prescribing. Finally, there may be a need to establish multidisciplinary professional WhatsApp groups that include dentists and medical professionals of different specialties such as medicine, surgery, obstetrics, pediatrics, etc., to ensure provision of the correct diagnosis and treatment.

\section{Authors' Contributions}

N.D.O., S.A.H., and O.A.H. conceptualized the study; S.A.H. and A.A. mapped out the methodology; A.A.H. and H.B. contributed in finding the resources; data curation was performed by H.B. and S.A.H.; N.D.O., A.A.H., and A.A. took part in original draft preparation of the article; O.A.H. performed review and editing of the manuscript; S.A.H. and A.A.H. supervised the entire study.

\section{Conflict of Interest}

None declared.

\section{Acknowledgment}

The authors also would like to thank Dr. Wisam Kherfan (Amman, Jordan), the admin of the participating WhatsApp groups for her efforts in facilitating the conduction of this study.

\section{References}

1 Odeh ND, Babkair H, Abu-Hammad S, Abu-Hammad A, Abu-Hammad O. COVID-19: present and future challenges for dental practice. Int J Environ Res Public Health 2020;17:3151

2 Du Y, Tu L, Zhu P, et al. Clinical features of 85 fatal cases of COVID-19 from Wuhan. A retrospective observational study. Am J Respir Crit Care Med 2020;201(11):1372-1379

3 American Dental Association. Available at: https://success. ada.org/ /media/CPS/Files/Open\%20Files/ADA_COVID19_ Dental_Emergency_DDS.pdf. Accessed June 20, 2020

4 Jampani ND, Nutalapati R, Dontula BS, Boyapati R. Applications of teledentistry: a literature review and update. J Int Soc Prev Community Dent 2011;1(2):37-44

5 Sykes L, Harryparsad A, Evans W, Gani F. Social media and dentistry: part 8: ethical, legal, and professional concerns with the use of internet sites by health care professionals. South African Dent J 2017;72(3):132-136

6 Lewis MAO. Why we must reduce dental prescription of antibiotics: European Union Antibiotic Awareness Day. Br Dent J 2008;205(10):537-538 
7 Dar-Odeh N, Ryalat S, Shayyab M, Abu-Hammad O. Analysis of clinical records of dental patients attending Jordan University Hospital: documentation of drug prescriptions and local anesthetic injections. Ther Clin Risk Manag 2008;4(5):1111-1117

8 Murti A, Morse Z. Dental antibiotic prescription in Fijian adults. Int Dent J 2007;57(2):65-70

9 Ramadan AM, Al Rikaby OA, Abu-Hammad OA, Dar-Odeh NS. Knowledge and attitudes towards antibiotic prescribing among dentists in Sudan. Pesqui Bras Odontopediatria Clin Integr 2019;19(1):e4430. doi:10.4034/PBOCl.2019.191.17

10 Dar-Odeh N, Fadel HT, Abu-Hammad S, Abdeljawad R, Abu-Hammad OA. Antibiotic prescribing for oro-facial infections in the paediatric outpatient: a review. Antibiotics (Basel) 2018;7(2):E38

11 Abdelrahim A, Shimpi N, Hegde H, et al. Feasibility of establishing tele-dental approach to non-traumatic dental emergencies in medical settings. Am J Dent 2020;33(1):48-52

12 Abu Hammad O, Alnazzawi A, Borzangy SS, et al. Factors influencing global variations in COVID-19 cases and fatalities; a review. Healthcare (Basel) 2020;8(3):E216

13 Spížek J, Řezanka T. Lincosamides: chemical structure, biosynthesis, mechanism of action, resistance, and applications. Biochem Pharmacol 2017;133:20-28

14 American Association of Endodontists. Endodontics and Antibiotic Update. Available at: https://f3f142zs0k2w1kg84k5p9i1o-wpengine.netdna-ssl.com/specialty/wp-content/ uploads/sites/2/2019/12/ecfe-fall-2019-REV11.pdf. Published 2019. Accessed May 16, 2020

15 Taiwo AO, Ibikunle AA, Braimah RO, Sulaiman OA, Gbotolorun OM. Tooth extraction: pattern and etiology from extreme Northwestern Nigeria. Eur J Dent 2017;11(3):335-339

16 Dar-Odeh NS, Al-Abdalla M, Al-Shayyab MH, et al. Prescribing antibiotics for pediatric dental patients in Jordan; knowledge and attitudes of dentists. Int Arab $\mathrm{J}$ Antimicrob Agents 2013;3(3):4

17 Oberoi SS, Dhingra C, Sharma G, Sardana D. Antibiotics in dental practice: how justified are we. Int Dent J 2015;65(1):4-10

18 Dar Odeh N, Binsaad SM, Gasim RA, et al. Why do women avoid dental visits during pregnancy? A cross- sectional survey in Al Madinah, Western Saudi Arabia. Pesqui Bras Odontopediatria Clin Integr 2018;18(1):e3934. Doi: 10.4034/ PBOCI.2018.181.31

19 Hammad OA, Binsaad SM, Gasim RA, et al. Assessing expectant mothers' knowledge and beliefs about oral healthcare during infancy and pregnancy: a cross sectional survey in Saudi Arabia. Pesqui Bras Odontopediatria Clin Integr 2018;18(1):e4027. Doi: 10.4034/PBOCI.2018.181.66

20 Henderson E, Mackillop L. Prescribing in pregnancy and during breast feeding: using principles in clinical practice. Postgrad Med J 2011;87(1027) :349-354

21 Donaldson M, Goodchild JH. Pregnancy, breast-feeding and drugs used in dentistry. J Am Dent Assoc 2012;143(8):858-871

22 Dubey S, Saha SG, Rajkumar B, Dhole TK. Comparative antimicrobial efficacy of selected root canal irrigants on commonly isolated microorganisms in endodontic infection. Eur J Dent 2017;11(1):12-16

23 Kazi MM, Bharadwaj R. Role of herpesviruses in chronic periodontitis and their association with clinical parameters and in increasing severity of the disease. Eur J Dent 2017;11(3):299-304

24 Dar-Odeh N, Shehabi A, Al-Bitar Z, et al. Oral Candida colonization in patients with fixed orthodontic appliances: the importance of some nutritional and salivary factors. Afr J Microbiol Res 2011;5(15):2150-2154

25 Gerber JS, Ross RK, Bryan M, et al. Association of broad- vs narrow-spectrum antibiotics with treatment failure, adverse events, and quality of life in children with acute respiratory tract infections. JAMA 2017;318(23):2325-2336

26 Jabra-Rizk MA, Kong EF, Tsui C, et al. Candida albicans pathogenesis: fitting within the host-microbe damage response framework. Infect Immun 2016;84(10):2724-2739

27 Stoopler ET, Ojeda D, Elmuradi S, Sollecito TP. Lymphoid hyperplasia of the tongue. J Emerg Med 2016;50(3):e155-e156

28 Chaux-Bodard AG, Deneuve S, Desoutter A. Oral manifestation of COVID-19 as an inaugural symptom? J Oral Med Oral Surg 2020;26:2(18)

29 Martín Carreras-Presas C, Amaro Sánchez J, López-Sánchez AF, Jané-Salas E, Somacarrera Pérez ML. Oral vesiculobullous lesions associated with SARS-CoV-2 infection [published online ahead of print, 2020 May 5]. Oral Dis 2020;10.1111/ odi.13382 doi:10.1111/odi.13382

30 Kotfis K, Skonieczna-Żydecka K. COVID-19: gastrointestinal symptoms and potential sources of SARS-CoV-2 transmission. Anaesthesiol Intensive Ther 2020;52(2):171-172

31 To KK-W, Tsang OT-Y, Yip CC, et al. Consistent detection of 2019 novel coronavirus in saliva. Clin Infect Dis 2020;71(15):841-843 